\title{
The implication of non-driving activities on situation awareness and take-over performance in level 3 automation
}

\author{
Lichao Yang \\ School of Aerospace Transport and \\ Manufacturing \\ Cranfield University \\ Bedford, UK \\ Lichao.yang@cranfield.ac.uk
}

\author{
Arkadiusz Dmitruk \\ School of Aerospace Transport and \\ Manufacturing \\ Cranfield University \\ Bedford, UK \\ Arkadiusz.Dmitruk@cranfield.ac.uk
}

\author{
Mahdi Babayi Semiromi \\ School of Aerospace Transport and \\ Manufacturing \\ Cranfield University \\ Bedford, UK \\ M.Babayi-Semiromi@cranfield.ac.uk \\ James Brighton \\ School of Aerospace Transport and \\ Manufacturing \\ Cranfield University \\ Bedford, UK \\ j.1.brighton@cranfield.ac.uk
}

\author{
Daniel Auger \\ School of Aerospace Transport and \\ Manufacturing \\ Cranfield University \\ Bedford, UK \\ d.j.auger@cranfield.ac.uk \\ Yifan Zhao \\ School of Aerospace Transport and \\ Manufacturing \\ Cranfield University \\ Bedford, UK \\ yifan.zhao@cranfield.ac.uk
}

\begin{abstract}
The driver's take-over performance is of great importance for driving safety in conditionally automated driving since the driver is required to respond appropriately to control the vehicle if there is a system failure. The engagement of different non-driving activities (NDAs), considered as the main factor of the driver's take-over performance has been investigated in this study from both perspectives of the driver's situation awareness and take-over quality. The activities are divided into 2 groups, which are active interaction mode and passive interaction mode based on the engagement of human and object. The results suggest that the engagement of NDAs could reduce the driver's situation awareness. Driver's attention level is different for each activity. Particularly, active interaction mode NDAs requests more mentally demanding and drivers are not sensitive to the driving situation change when they are doing such activities. In addition, there is no significant difference in the maximum lateral error with NDAs engagement. However, it takes more time to achieve a safe control transition for drivers who are doing the NDAs. The active interaction mode NDAs request even more time. Moreover, the transition process could benefit from steering wheel haptic feedback torque, which can be considered as an effective take-over assistance system.
\end{abstract}

Keywords-automated driving, attention level, haptic torque, Non-driving-related-task (NDRT), driver behaviour, transition of control

\section{INTRODUCTION}

A highly automated driving vehicle could free the driver's eye and hand from controlling the vehicle in some driving scenarios. It could encourage the driver to engage some nondriving activities (NDAs) [1], [2]. However, fully automated driving has not been achieved yet. Automated driving vehicles can not provide an appropriate response for every driving scenario, which is a potential safety risk and a main concern of the current automated driving system [3]. According to the SAE (J3016) Automation Levels [4], in Level 3 automation, the driver only needs to control the vehicle when the intervene is requested, which means the driver could engage some NDAs rather than pay full attention to driving under the automated driving mode. Since the engagement of NDAs could reduce the driver's situation awareness and attention [5]-[7], it is of great importance to evaluate its impact on the take-over performance to achieve a safe and smooth control transition.

Researches suggested that the sufficient take-over interval for drivers should be 5 to 8s [7], [8]. It is affected by different factors such as driver's state including age, gender, driving experience [9], [10], the complexity of the driving scenario [11]-[13], the modality of the take-over request [8], [14], [15] and the NDAs that drivers engage with [8], [16]. The impact of diverse NDAs on take-over performance has been widely researched in recent years. Yooh et al. [8] investigated the driver's take-over performance with 3 types of NDA, which are phone conversation, smartphone interaction, and video watching tasks, while Zeeb et al. [1] examined the impact of writing an email, reading news, and watching a video clip. Results from both studies suggested that the NDA engagement can significantly influence the take-over quality based on the statistical analysis. One of the limitations of existing studies [17], [18] is that NDAs were investigated specifically and independently, which limits the extendibility of the driver monitoring or take-over assistance system. When considering a new NDA, such a system needs to conduct the evaluation process again to investigate its impact. There is a lack of a systematic method to group or categorise NDAs which could have a similar level of impact on the take-over performance. On the other hand, the existing literature of NDA's impact is normally from the perspective of the driver's workload [3], [7], [19]. The situation awareness before take-over is also considered as a crucial factor of safe take-over transition but has not been discussed associated with NDAs [20]. There is limited literature that attempted to investigate the implication of situation awareness on the take-over process. The evaluation of the driver's situation awareness can be further used to predict the driver's road-checking behaviour and takeover performance.

The existing literature has claimed that the driver's takeover performance is affected by the type of NDAs. For instance, visual related activities tend to take a longer reaction time than auditory related activities [21]. However, the number of evaluated NDAs is limited. Following the survey made by Sivak and Schoettle [22], the common NDAs are reading, texting, working, watching movies and playing games. In this study, we picked 4 types of visual-related NDA 


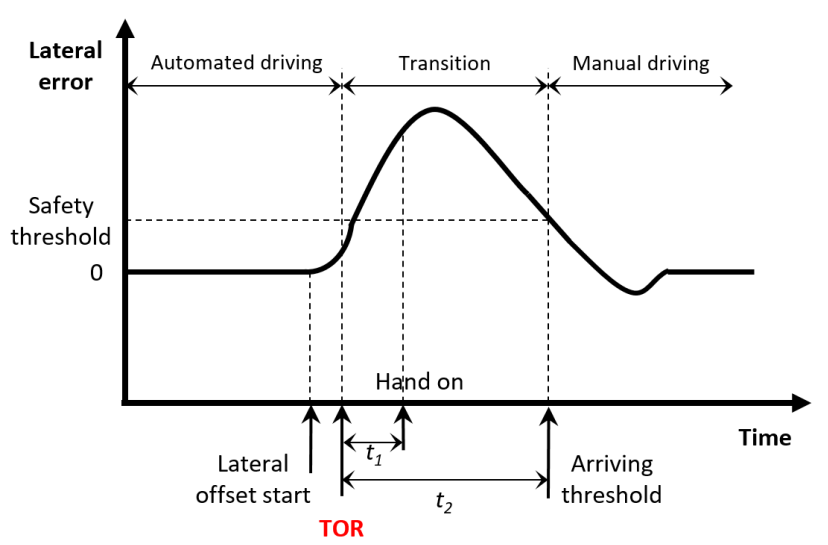

Fig. 1. Concept of the take-over process

which are playing games, answering questionnaires, watching videos and reading news and further evaluated them on the same device which is a tablet. Based on the way of interaction between human and object, the NDAs are divided into 2 groups, which are active interaction mode and passive interaction mode. Playing games and answering questionnaires can be considered as active interaction mode since the driver and the object respond to each other's action overtime during the engagement. However, under passive interaction mode like reading news or watching movies, the driver only receives information passively. This study hypothesises that the workload and demanded attention are different between these two modes. Furthermore, compared with the passive mode NDAs, the active mode NDAs could result in a more negative impact on the driver's take-over performance.

This paper investigates the implication of NDAs in different interaction modes on the take-over performance in level 3 automation. Furthermore, the driver's behaviour has been recorded including hand and gaze movement, which is used to evaluate the driver's road-checking behaviour and infer its motivation associated with NDAs. To ensure the safe take-over transition, the haptic feedback has been added on the steering wheel. The haptic feedback in the human-machine interface (HMI) design for the take-over process has been widely researched [23]-[25], specifically implemented on the steering wheel [26]-[28]. In this study, the effectiveness and impact of haptic feedback in take-over performance are also evaluated. The vehicle setting and the experiment design are introduced in Section II. In Section III, the driver's roadchecking behaviour and take-over performance of each NDAs are evaluated and discussed in both group and individual levels. Discussion and conclusion are given in Section IV.

\section{Methodology}

\section{A. Experiment Design}

The design of the take-over process in a trial is illustrated in Fig. 1. During a trial, the vehicle was driving automatically initially while the participant was required to do a type of NDA or checking the road. Then the take-over process started after a lateral offset implemented to the vehicle. The lateral error is defined as the distance between the vehicle position and the closest point on the path. After a lateral offset implemented, the vehicle is on an improper position of the road, an acoustic signal as a take-over request (TOR) was then given to the participant. The participant was requested to take control of the vehicle and bring it back to the right position. In Fig. 1, $t_{1}$ indicates the time needed for the driver

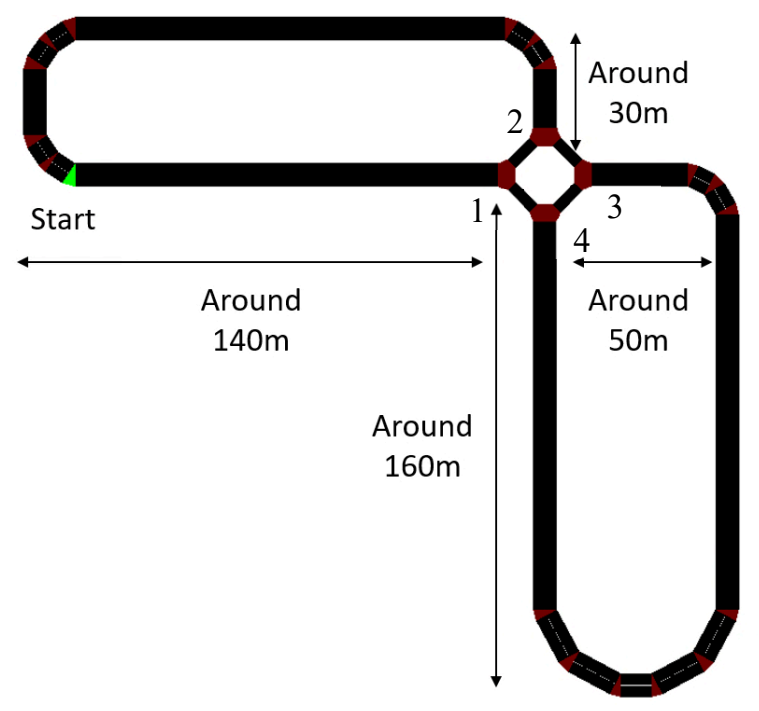

Fig. 2. Sketch map of the track. putting her/his hand on the steering wheel. To achieve a safe and smooth take-over transition, a haptic torque was implemented to help the driver guide the vehicle to the reference route. The haptic torque was engaged as soon as the driver applies torque to the wheel and gradually fades away. After the lateral error achieves the maximum value, the vehicle will return to the reference route. A threshold of the safety distance is defined, which indicates the control transition is finished and the driver could achieve a safe manual driving afterwards. In this study, the threshold was set as $1 \mathrm{~m}$. In Fig. 1, $t_{2}$ refers to the time needed from TOR to the time when the vehicle arrives at the threshold, which is considered as a criterion to evaluate the take-over performance in this study.

\section{B. Experimental Platform}

1) Vehicle Modification: The vehicle used for the experiments was an instrumented Landrover Discovery 5. The car was modified to accommodate both autonomous and human driving. An electric motor, operating on the steering column, was used for steering and another electric motor was used to control the throttle pedal position. Braking was modified using a pneumatic actuator on the brake pedal. To ensure the safety, a steering wheel and a set of pedals were added in the back seat, which allows a safety driver to intervene and override the autonomous system. For path following, the pure pursuit algorithm was used to generate the reference steering angle. The rear steering wheel was controlled using the reference steering angles and the front wheel follows the rear wheel.

2) Participants: A total of 16 participants (14 male and 2 female) from Cranfield University were recruited for this experiment. The participants' age is in a range from 24 to 30 . They were required to hold a valid UK driving license while they have no driving experience of automated vehicles.

3) NDAs: Four types of NDA are investigated in this study, which include reading news, watching videos, playing games and answering questionnaires using a tablet. For reading, the participant was required to read some articles from BBC News. For watching videos, the participant was asked to watch Youtube videos. Temple Run was used as the target game for the game engagement. For the NDA of answering questionnaires, the participant was required to complete a questionnaire, which comprises some objective 


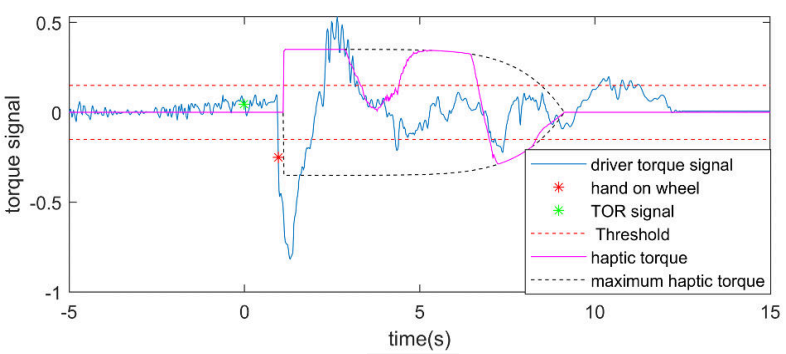

(a)

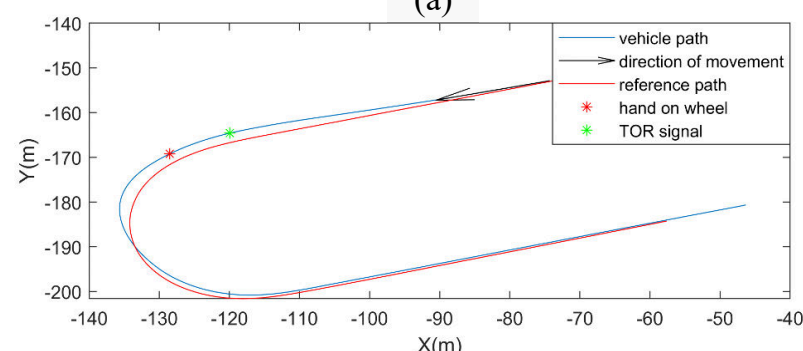

(b)

Fig. 3. Top plot presents the driver's torque and the haptic torque for 1 instance. Bottom plot presents the corresponding vehicle movement in the track.

and subjective questions about this experiment. In the experiment, there were 7 trials per participant. It includes 4 trials for 4 types of NDA respectively and 1 trial without NDA (watching road). For the remaining 2 trials, 2 activities were randomly selected from the 5 activities mentioned above. The order of each activity was randomly selected to reduce the bias.

4) Track and Take-over Scenarios: The testing track is a two-lane road with a mini-roundabout, as shown in Fig. 2. The start point is highlighted with green colour. In the odd loop, the vehicle enters from the exit 1 into the miniroundabout and leaves from the exit 3 . Then it enters from the exit 4 and leaves from the exit 2 . In the even loop, the vehicle enters from the exit 1 and leaves the mini-roundabout from the exit 4 . Then it enters into the roundabout from the exit 3 and leaves from the exit 2 . The TOR signal was issued at specific points on the track to avoid the area around the mini roundabout for the safety concern. The lateral offset was set as $1.5 \mathrm{~m}$ with a small variation in the real trial. The maximum speed of the vehicle was set as $30 \mathrm{mph}$. The interval between TORs was randomly selected from the range of 5 to 9 minutes.

\section{Data Acquisition}

An OXTS RT1003 with RTK GPS was used for positioning and a dSPACE Microautobox I was used as an onboard computer. The RT1003 system provides the global vehicle position with an accuracy of $2 \mathrm{~cm}$ and the heading angle with an accuracy of less than 1 degree. The data of vehicle status were recorded in the Micorautobox I at a

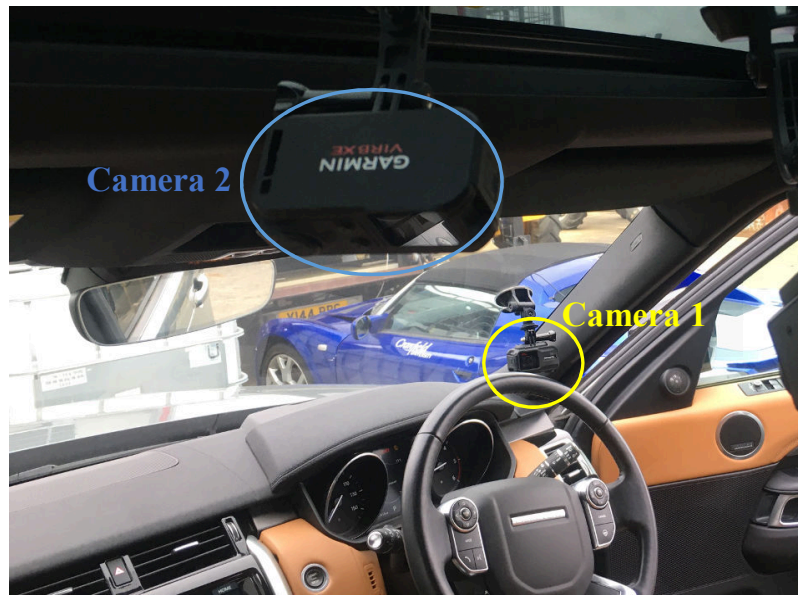

Fig. 4. Cameras inside the vehicle. Locations have been highlighted by different colours.

sampling rate of $1 \mathrm{kHz}$. The data include driver steering torque, autonomous steering torque, vehicle position and heading, vehicle velocity, steering angle and take-over signal. The path was recorded beforehand at $1 \mathrm{kHz}$ and then resampled by the linear interpolation to a spatial accuracy of $0.2 \mathrm{~m}$.

Driver's hand-on-wheel time $\left(t_{1}\right)$ was defined as the moment that the driver's applied torque passes a certain threshold. The threshold was experimentally determined to avoid false take-over detection due to sensor noise. An instance of the driver's torque during a take-over process is shown in Fig. 3(a). The corresponding vehicle route is presented in Fig. 3(b).

After the driver takes control of the steering wheel, the vehicle provides haptic cues to the driver, in the form of torque on the steering wheel, to increase driver's awareness of the environment. The haptic decays over a certain amount of time and eventually reaches 0 to give the driver full control. The value of the torque is calculated using

$$
\tau_{\text {haptic }}(t)=K_{t}(t) K_{p}\left(\delta-\delta_{\text {ref }}\right)
$$

where $\delta$ is the vehicle steering angle; $\delta_{\text {ref }}$ is the reference steering angle calculated by the path following algorithm; $K_{p}$ is a constant gain and $K_{t}(t)$ is a decaying gain which is a function of time starting from 1 and reaching to 0 at the end of the take-over period. The decaying profile is shown in Fig. 3(a). The decaying duration chosen for this experiment was 8 seconds. The torque value is normalised between -1 and 1 , where 1 indicates the maximum torque of the electric motor in one direction and -1 indicates the maximum torque in another direction. The maximum amplitude of the torque was a tuning parameter. Each participant tried two of three pre-set values: $0.35,0.45,0.55$.

There were 2 cameras (Garmin Virb Action Camera) employed to monitor the driver's behaviour during the experiment. The resolution of both cameras was set as $1920 \times$ 1440 pixels and images were sampled at 24 frames per second

TABLE I. ROAD-CHECKING BEHAVIOUR EVALUATION

\begin{tabular}{|c|c|c|c|c|c|}
\hline \multirow{2}{*}{ NDAs } & \multirow{2}{*}{$\begin{array}{l}\text { Checking } \\
\text { period (s) }\end{array}$} & \multicolumn{4}{|c|}{ Percentage of checking for corresponding motivation } \\
\hline & & Bumping & Approaching junctions & Breakpoint & Others \\
\hline Watching videos & 37.10 & $19.88 \%$ & $52.05 \%$ & $5.85 \%$ & $22.22 \%$ \\
\hline Reading news & 51.64 & $16.78 \%$ & $51.75 \%$ & $7.69 \%$ & $23.78 \%$ \\
\hline Answering questionnaires & 123.00 & $18.18 \%$ & $50.00 \%$ & $13.64 \%$ & $18.18 \%$ \\
\hline Playing games & 79.13 & $3.61 \%$ & $26.50 \%$ & $59.04 \%$ & $10.84 \%$ \\
\hline
\end{tabular}




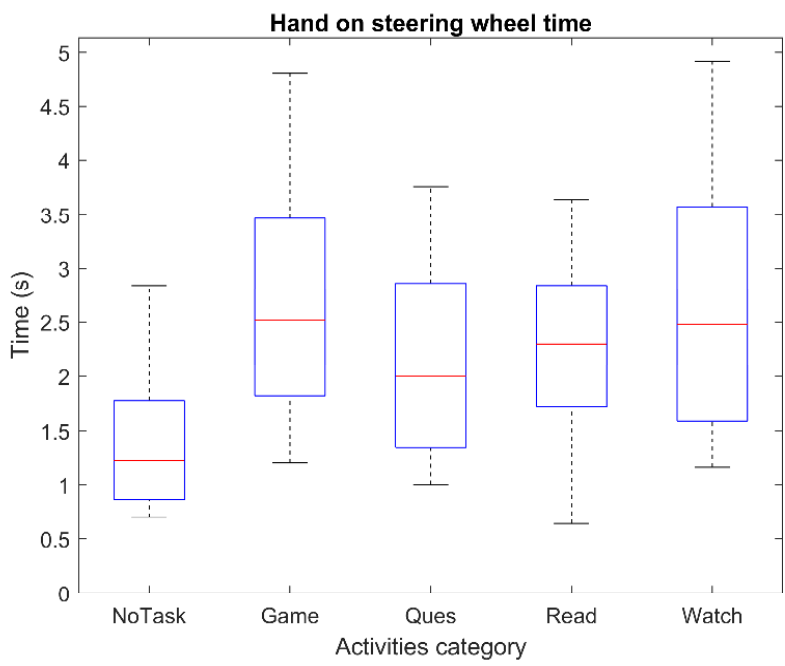

Fig. 5. The hand-on-wheel time performance. Ques refers to the answering questionnaires.

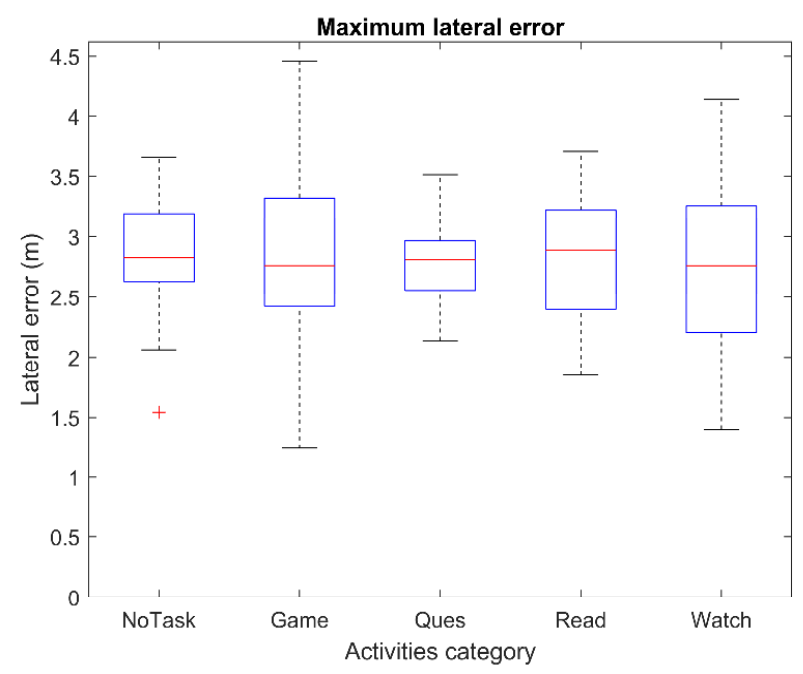

Fig. 6. Maximum lateral error achieveing

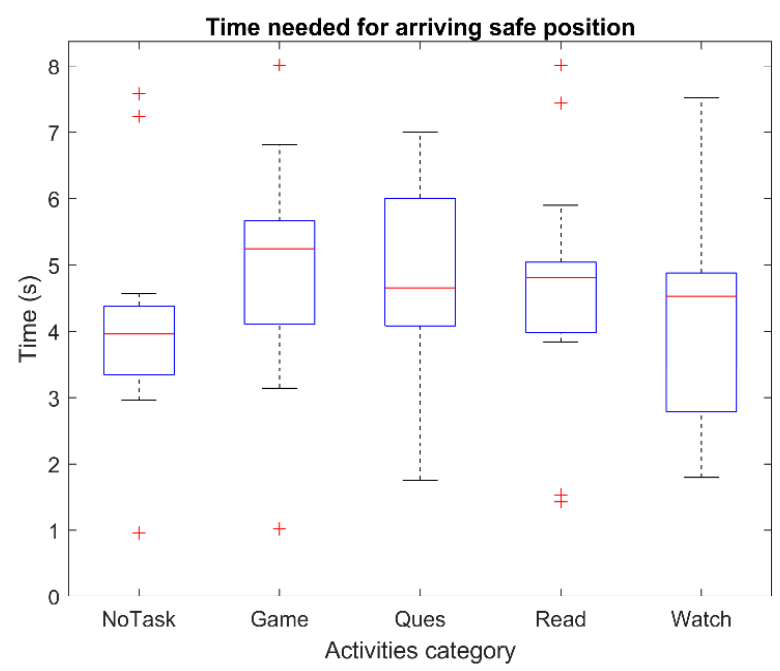

Fig. 7. Time cost for the vehicle back to the safe position

(fps). As shown in Fig. 4, one camera (Camera 1) was located in the right bottom of the windscreen and faced to the driver's face, which is used to detect whether the driver's gaze is engaging with NDAs or checking the road. Another one (Camera 2) was mounted on the roof of the vehicle between two front seats to record the driver's hand movement engaging with the tablet or steering wheel. The following analysis of situation awareness is based on the process of the recorded videos. The video clip captured from Camera 1 was used to evaluate if the driver conducts road-checking behaviours. The inferred motivation was manually labelled based on the videos recorded from Camera 2.

\section{RESULTS}

\section{A. Situation awareness}

The statistic results of driver road-checking behaviour for all participants are presented in Table I. The checking period is calculated by the duration of the NDA trial and the total number of checking behaviour in this trial. The motivation for road-checking behaviour is inferred by reviewing the videos from two cameras. Bumping refers to the vehicle vibration due to uneven road surface. For approaching junctions, the driver's glance is counted when approaching the roundabout and turning. Breakpoint indicates the roadchecking behaviour due to a short break during the NDA engagement. For instance, the driver sometimes checks the environment after she/he finishes watching a video clip or a round of game. Other covers the road-checking behaviour without unclear motivation or regular road-checking.

It has been observed that the checking period is lowest (37.1s) when the driver was watching videos. For this NDA, the main motivations of road-checking are Approaching junction (52.05\%) and Bumping (19.88\%). Reading news has the second-lowest period $(51.64 \mathrm{~s})$, where the proportion of motivations is similar to that of watching videos. Answering questionnaires has the least road-checking behaviour. Normally only once or twice in a trial. Approaching junction $(50 \%)$ still dominates the motivation. As one of the typical NDAs under the active interaction mode, playing games has a relatively high road-checking period $(79.13 \mathrm{~s})$, where Breakpoint (59.04\%) dominates the motivation. The proportion of Approaching junctions and Bumping are 26.5\% and $3.61 \%$, respectively. Compared to the NDAs in active interaction mode, the NDAs in passive interaction mode leads to more frequent road-checking, which suggests drivers have more awareness for the situations of vehicle vibration, turning or slowing down when approaching junctions. These road-checking behaviours are important to ensure a safe transition if the take-over is required under these scenarios. The observation also suggests that the driver has a relatively low workload under passive interaction mode, which potentially leads to a more smooth and better quality takeover process. For the NDAs under active interaction mode, the results show that the driver paid a high level of engagement on the activity, particularly for answering questionnaires, evident by much less frequent road-checking. For playing games, the road-checking normally happens during Breakpoint and the driver is not sensitive to the driving-situation change during a game. Therefore, for this type of NDA, the driver is more difficult to complete a highquality take-over transition due to lack of situation awareness.

\section{B. Take-over performance}

The driver's performance during the take-over process is presented in this section. The driver's hand-on-wheel time $\left(t_{1}\right)$ is shown in Fig. 5. As expected, no NDA engagement achieved the shortest $t_{1}$ with an average value around $1.2 \mathrm{~s}$. 
TABLE II. TAKE-OVER PERFORMANCE EVALUATION OF ACTIVITIES

\begin{tabular}{|c|r|r|r|r|r|}
\hline \multirow{2}{*}{$\begin{array}{c}\text { Time to } \\
\text { Threshold }\end{array}$} & \multicolumn{5}{|c|}{ Activities } \\
\cline { 2 - 6 } & No task & Watch & \multicolumn{1}{c|}{ Read } & \multicolumn{1}{c|}{ Ques } & Game \\
\hline Mean (s) & 4.08 & 4.19 & 4.68 & 4.82 & 4.98 \\
\hline $\begin{array}{c}\text { Standard } \\
\text { deviation (s) }\end{array}$ & 1.60 & 1.48 & 1.59 & 1.38 & 1.52 \\
\hline
\end{tabular}

TABLE III. TAKE-OVER PERFORMANCE EVALUATION OF HAPTIC TORQUE LEVELS

\begin{tabular}{|c|r|r|r|}
\hline \multirow{2}{*}{$\begin{array}{c}\text { Time to } \\
\text { Threshold }\end{array}$} & \multicolumn{3}{|c|}{ Haptic torque level } \\
\cline { 2 - 4 } & Low & \multicolumn{1}{c|}{ Medium } & \multicolumn{1}{c|}{ High } \\
\hline Mean (s) & 4.81 & 4.68 & 4.38 \\
\hline $\begin{array}{c}\text { Standard } \\
\text { deviation (s) }\end{array}$ & 1.54 & 1.23 & 1.64 \\
\hline
\end{tabular}

For the selected 4 NDAs, the average $t_{1}$ is in the range of 2$2.5 \mathrm{~s}$, which is more than double than without NDA. Playing games seems to result in the longest $t_{1}$. From Fig. 6, it can be observed the maximum lateral error for each activity is similar, which is in the range of 2.5 to $3.5 \mathrm{~m}$. In most of the trials, after receiving the TOR signal, the driver can obtain the control of the vehicle and prevent the situation from getting worse within a $3.5 \mathrm{~m}$ lateral error. However, NDA engagement affects the driver's controlling performance after the vehicle achieving the maximum lateral error. It can be seen from Fig. 7 that the time needed to arrive the safe position without NDA engagement $\left(t_{2}\right)$ is around $4 \mathrm{~s}$, while for all types of NDA engagement $t_{2}$ is at least $0.5 \mathrm{~s}$ more, which suggests that the vehicle could stay in a dangerous position for a longer time. Mean and standard deviation of $t_{2}$ for each activity are presented in Table II. The mean values of watching videos and reading news (passive interaction mode) are $4.19 \mathrm{~s}$ and $4.68 \mathrm{~s}$, representatively, which are higher than those of answering questionnaires and playing games (4.82s and $4.98 \mathrm{~s}$ respectively) in active interaction mode. Again, playing games leads to the longest $t_{2}$. There is no significant difference between watching videos and no NDA engagement. Through combining Fig. 5 and Fig. 7, it has been suggested that NDAs in active interaction mode request more time to control the vehicle during the take-over process. The reason could be that for this type of NDA the driver needs more time to develop the awareness of driving-environment after receiving the TOR signal and is more difficult to recover from the previous NDA mentally.

The take-over performance of haptic feedback is presented in Table III. For a low level of haptic torque, the mean and standard deviation of $t_{2}$ are $4.81 \mathrm{~s}$ and $1.54 \mathrm{~s}$. With the increase of the haptic torque level, the mean value of $t_{2}$ decreases, which suggests that a higher level of haptic feedback could decrease $t_{2}$ and improve take-over performance.

\section{CONCLUSION}

In level 3 automated driving, one of the most important challenges for driving safety is the take-over process. It is affected by many factors but dominated by the driver's state before take-over. This study investigated the implication of four selected NDAs, grouped into active and passive interaction modes, on situation awareness during the NDA engagement associated with its motivation and the following take-over performance. The approach of grouping aims to extend the application of this study on a wide range of NDA. Furthermore, the effectiveness of steering wheel haptic assistance system for the take-over process has been evaluated.

From the situation awareness point of view, drivers always check the environment to ensure driving safety during the NDAs engagement. Compared to the NDAs in active interaction mode, the NDAs in passive interaction mode leads to more frequent road-checking, which suggests drivers have more awareness for the situations of vehicle vibration, turning or slowing down when approaching junctions. The motivation study also suggests that for the NDAs in active interaction mode the driver is not sensitive to the driving situation change. Drivers should be warned when they engage with this kind of NDA and do not check the road for a long period.

For the take-over process, the engagement of NDAs could result in a negative effect. It has been observed that the driver who engages with NDAs in active interaction mode requests more time to achieve a safe take-over transition. Moreover, a higher level of haptic feedback could decrease $t_{2}$ and improve take-over performance.

In summary, the type of NDA determines the level of demanded attention of the driver, which influences the situation awareness and take-over quality. The observed results also suggest that the take-over process could benefit from the high-frequency road-checking and haptic feedback assistance. The investigation of these factors helps us develop a deep understanding of the implication of human behaviour on the take-over performance, which could help for further take-over strategy and HMI design to achieve the safe control transition.

\section{ACKNOWLEDGMENT}

This work was partly supported by Jaguar Land Rover and the UK-EPSRC grant EP/N012089/1 as part of the jointly funded Towards Autonomy: Smart and Connected Control (TASCC) Programme; partly supported by Cranfield's EPSRC Impact Accrelate Account EP/R511511/1.

\section{REFERENCES}

K. Zeeb, A. Buchner, and M. Schrauf, "Is take-over time all that matters? The impact of visual-cognitive load on driver take-over quality after conditionally automated driving," Accid. Anal. Prev., vol. 92, pp. 230-239, Jul. 2016.

[2] L. Yang, K. Dong, A. J. Dmitruk, J. Brighton, and Y. Zhao, “A Dual-Cameras-Based Driver Gaze Mapping System With an Application on Non-Driving Activities Monitoring," IEEE Trans. Intell. Transp. Syst., pp. 1-10, 2019

[3] D. Choi, T. Sato, T. Ando, T. Abe, M. Akamatsu, and S. Kitazaki, "Effects of cognitive and visual loads on driving performance after take-over request (TOR) in automated driving," Appl. Ergon., vol. 85, no. February, p. 103074, May 2020 .

[4] Taxonomy and Definitions for Terms Related to Driving Automation Systems for On-Road Motor Vehicles, SAE International Standard J3016_201806, 2018. "Effects of adaptive cruise control and highly automated driving 
on workload and situation awareness: A review of the empirical evidence," Transp. Res. Part F Traffic Psychol. Behav., vol. 27 , no. PB, pp. 196-217, Nov. 2014.

E. Dogan, M.-C. Rahal, R. Deborne, P. Delhomme, A. Kemeny, and J. Perrin, "Transition of control in a partially automated vehicle: Effects of anticipation and non-driving-related task involvement," Transp. Res. Part F Traffic Psychol. Behav., vol. 46, pp. 205-215, Apr. 2017.

S. H. Yoon and Y. G. Ji, "Non-driving-related tasks, workload, and takeover performance in highly automated driving contexts," Transp. Res. Part F Traffic Psychol. Behav., vol. 60, pp. 620631, Jan. 2019.

[8] S. H. Yoon, Y. W. Kim, and Y. G. Ji, "The effects of takeover request modalities on highly automated car control transitions," Accid. Anal. Prev., vol. 123, no. November 2018, pp. 150-158, Feb. 2019.

[9] H. Clark and J. Feng, "Age differences in the takeover of vehicle control and engagement in non-driving-related activities in simulated driving with conditional automation," Accid. Anal. Prev., vol. 106, pp. 468-479, Sep. 2017.

[10] S. Li, P. Blythe, W. Guo, and A. Namdeo, "Investigating the effects of age and disengagement in driving on driver's takeover control performance in highly automated vehicles," Transp. Plan. Technol., vol. 42, no. 5, pp. 470-497, Jul. 2019.

[11] J. Radlmayr, C. Gold, L. Lorenz, M. Farid, and K. Bengler, "How Traffic Situations and Non-Driving Related Tasks Affect the Take-Over Quality in Highly Automated Driving," Proc. Hum. Factors Ergon. Soc. Annu. Meet., vol. 58, no. 1, pp. 2063-2067, Sep. 2014.

[12] M. S. L. Scharfe, K. Zeeb, and N. Russwinkel, "The Impact of Situational Complexity and Familiarity on Takeover Quality in Uncritical Highly Automated Driving Scenarios," Information, vol. 11, no. 2, p. 115, Feb. 2020.

[13] C. Wu, H. Wu, N. Lyu, and M. Zheng, "Take-Over Performance and Safety Analysis Under Different Scenarios and Secondary Tasks in Conditionally Automated Driving," IEEE Access, vol. 7, pp. 136924-136933, 2019.

[14] B. Wandtner, N. Schömig, and G. Schmidt, "Effects of NonDriving Related Task Modalities on Takeover Performance in Highly Automated Driving," Hum. Factors J. Hum. Factors Ergon. Soc., vol. 60, no. 6, pp. 870-881, Sep. 2018.

[15] H. Jeong and Y. Liu, "Effects of non-driving-related-task modality and road geometry on eye movements, lane-keeping performance, and workload while driving," Transp. Res. Part F Traffic Psychol. Behav., vol. 60, pp. 157-171, Jan. 2019.

[16] J. Kim, H.-S. Kim, W. Kim, and D. Yoon, “Take-over performance analysis depending on the drivers' non-driving secondary tasks in automated vehicles," in 2018 International Conference on Information and Communication Technology Convergence (ICTC), 2018, pp. 1364-1366.
[17] S. Petermeijer, F. Doubek, and J. de Winter, "Driver response times to auditory, visual, and tactile take-over requests: A simulator study with 101 participants," in 2017 IEEE International Conference on Systems, Man, and Cybernetics (SMC), 2017, vol. 2017-Janua, pp. 1505-1510.

[18] H. Kim, W. Kim, J. Kim, and D. Yoon, "A Study on the Control Authority Transition Characteristics by Driver Information," in 2019 International Conference on Computational Science and Computational Intelligence (CSCI), 2019, pp. 1562-1563.

[19] M. Bueno, E. Dogan, F. Hadj Selem, E. Monacelli, S. Boverie, and A. Guillaume, "How different mental workload levels affect the take-over control after automated driving," in 2016 IEEE 19th International Conference on Intelligent Transportation Systems (ITSC), 2016, pp. 2040-2045.

[20] L. Petersen, L. Robert, X. J. Yang, and D. Tilbury, "Situational Awareness, Driver's Trust in Automated Driving Systems and Secondary Task Performance," SAE Int. J. Connect. Autom. Veh., vol. 2, no. 2, pp. 12-02-02-0009, May 2019.

[21] B. Wandtner, G. Schmidt, N. Schoemig, and W. Kunde, "Nondriving related tasks in highly automated driving - Effects of task modalities and cognitive workload on take-over performance," in AmE 2018 - Automotive meets Electronics; 9th GMMSymposium, 2018, pp. 1-6.

[22] M. Sivak, B. Schoettle, "Motion Sickness in Self-Driving Vehicles," Transportation Res. Inst., Ann Arbor, Univ. Michigan, Ann Arbor, MI, USA, Tech. Rep. UMTRI-2015-12, Apr. 2015.

[23] S. M. Petermeijer, D. A. Abbink, M. Mulder, and J. C. F. de Winter, "The Effect of Haptic Support Systems on Driver Performance: A Literature Survey," IEEE Trans. Haptics, vol. 8, no. 4, pp. 467-479, Oct. 2015.

[24] J. Wan and C. Wu, "The Effects of Vibration Patterns of TakeOver Request and Non-Driving Tasks on Taking-Over Control of Automated Vehicles," Int. J. Human-Computer Interact., vol. 34, no. 11, pp. 987-998, Nov. 2018.

[25] C. Lv et al., "Characterization of Driver Neuromuscular Dynamics for Human-Automation Collaboration Design of Automated Vehicles," IEEE/ASME Trans. Mechatronics, vol. 23, no. 6, pp. 2558-2567, Dec. 2018.

[26] S. M. Petermeijer, J. C. F. de Winter, and K. J. Bengler, "Vibrotactile Displays: A Survey With a View on Highly Automated Driving," IEEE Trans. Intell. Transp. Syst., vol. 17, no. 4, pp. 897-907, Apr. 2016.

[27] C. Lv et al., "A Novel Control Framework of Haptic Take-Over System for Automated Vehicles," in 2018 IEEE Intelligent Vehicles Symposium (IV), 2018, vol. 2018-June, no. Iv, pp. 15961601.

[28] C. Lv et al., "Human-Machine Collaboration for Automated Vehicles via an Intelligent Two-Phase Haptic Interface," arXiv, Feb. 2020. 
The implication of non-driving activities on situation awareness and take-over performance in level 3 automation

\author{
Yang, Lichao
}

IEEE

Yang L, Semiromi MB, Auger D,et al., (2021) The implication of non-driving activities on situation awareness and take-over performance in level 3 automation. In: IECON 2020 46th Annual Conference of the IEEE Industrial Electronics Society, 19-21 October 2020, Singapore https://doi.org/10.1109/IECON43393.2020.9254533

Downloaded from Cranfield Library Services E-Repository 